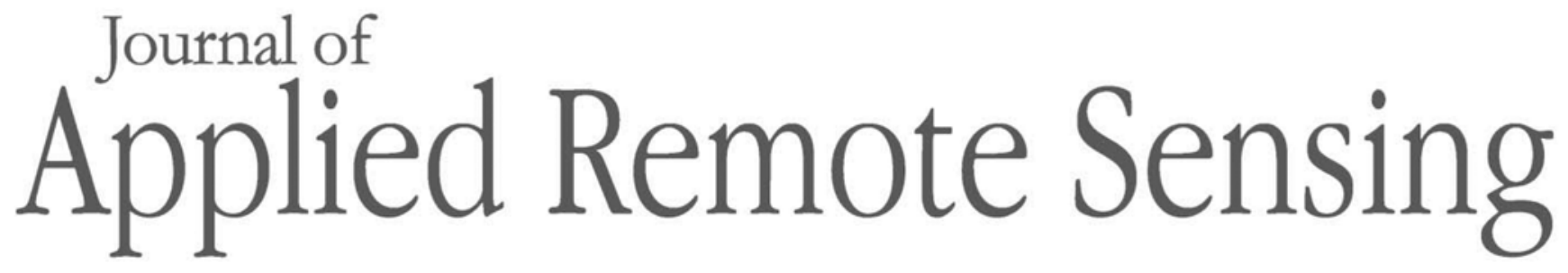

RemoteSensing.SPIEDigitalLibrary.org

\title{
Validation of MODIS-based monitoring for a green tide in the Yellow Sea with the aid of unmanned aerial vehicle
}

Fuxiang $\mathrm{Xu}$

Zhiqiang Gao

Weitao Shang

Xiaopeng Jiang

Xiangyu Zheng

Jicai Ning

Debin Song

Fuxiang Xu, Zhiqiang Gao, Weitao Shang, Xiaopeng Jiang, Xiangyu Zheng, Jicai Ning, Debin Song, "Validation of MODIS-based monitoring for a green tide in the Yellow Sea with the aid of unmanned aerial vehicle," J. Appl. Remote Sens. 11(1), 012007 (2017), doi: 10.1117/1.JRS.11.012007. 


\title{
Validation of MODIS-based monitoring for a green tide in the Yellow Sea with the aid of unmanned aerial vehicle
}

\author{
Fuxiang Xu, ${ }^{\text {a,b }}$ Zhiqiang Gao, ${ }^{\text {a,* }}$ Weitao Shang, ${ }^{\text {a }}$ Xiaopeng Jiang, ${ }^{\text {a }}$ \\ Xiangyu Zheng, ${ }^{\text {a,b }}$ Jicai Ning, ${ }^{a}$ and Debin Songa,b \\ ${ }^{a}$ Chinese Academy of Sciences, Yantai Institute of Coastal Zone Research, Yantai, China \\ ${ }^{b}$ University of Chinese Academy of Sciences, Beijing, China
}

\begin{abstract}
Previous studies have shown that Terra moderate resolution imaging spectroradiometer (MODIS) has low detection and characterization efficiency when mapping a green tide (Ulva prolifera) in the Yellow Sea. To quantify the uncertainty in mapping of the green tide using MODIS data, comparisons were conducted between quasi synchronous MODIS images and in situ observation data, as well as an unmanned aerial vehicle (UAV) image. The results show that MODIS images could detect the location of large $(>100 \mathrm{~m})$ floating green algae patches with good positional accuracy but tended to ignore the existence of small patches less than $10 \mathrm{~m}$ in width. The floating macroalgae area extracted using MODIS was several times larger than the area mapped using the UAV image. The Sentinel-2 multispectral instrument, the Chinese highresolution GF-1 wide field camera, and the Chinese HJ-1 charge-coupled device are recommended for early green tide detection, whereas MODIS is suitable for green tide monitoring. The UAV could also play an important role in regional green tide monitoring with the advantages of flexibility, smaller dimensions, high spatial resolution, and low cost. (c) 2017 Society of PhotoOptical Instrumentation Engineers (SPIE) [DOI: 10.1117/1.JRS.11.012007]
\end{abstract}

Keywords: green tide; MODIS; validation; unmanned aerial vehicle; research vessel.

Paper 16807SS received Nov. 13, 2016; accepted for publication Mar. 21, 2017; published online Mar. 29, 2017.

\section{Introduction}

Since 2008, a large-scale green tide disaster has occurred in the Yellow Sea due to the impact of fast-growing macroalgae, especially Ulva prolifera. ${ }^{1}$ Millions of tons of Ulva macroalgae, covering an area of thousands of square kilometers, floated on the sea surface during the recurrent phenomenon. ${ }^{2}$ Noxious odors $\left(\mathrm{NH}_{3}, \mathrm{H}_{2} \mathrm{~S}\right)$ and anoxic conditions caused by algal decay processes result in a considerable reduction of cultured fish and shrimp and even cause marine organism fatalities. ${ }^{3,4}$ In addition, masses of Ulva also impact the coastal tourism industry, thus significantly influencing the coastal economy, ecological environment, and aquaculture industry. ${ }^{5-9}$

Due to the extensive spatial distribution of the green tide, traditional monitoring is inadequate for conducting routine and real-time monitoring. Due to its timeliness, economic efficiency, and ability to survey large areas simultaneously, ${ }^{10,11}$ satellite remote sensing instruments, such as the sea-viewing wide field-of-view sensor, medium resolution imaging spectrometer, moderate resolution imaging spectroradiometer (MODIS), hyperspectral, Landsat multispectral scanner, thematic mapper, and enhanced thematic mapper, have been highly utilized in mapping floating cyanobacterial scum and seagrasses that have the similar characteristics as Ulva. ${ }^{12-17}$

In particular, MODIS images have been widely applied to green tide monitoring due to its availability and temporal resolution (twice a day). ${ }^{18} \mathrm{Wu}$ et al. ${ }^{19}$ extracted the bloom timing and areal coverage of green tides in the Shandong Peninsula from 2008 to 2012 by combining MODIS data with the normalized difference vegetation index (NDVI). A comprehensive comparison determined that the green tide exploded sharply in 2008 and 2009 compared to other

*Address all correspondence to: Zhiqiang Gao, E-mail: zqgao@yic.ac.cn

$1931-3195 / 2017 / \$ 25.00$ C 2017 SPIE 
years. ${ }^{19} \mathrm{Hu}^{20}$ proposed the floating algae index (FAI) and monitored the sea area near Qingdao, compared the results with NDVI, and proposed that FAI can help extract green tide information from satellite images. Keesing et al. ${ }^{21}$ and Liu et al. ${ }^{22}$ extracted the Yellow Sea green tide bloom information from 2007 to 2009 using MODIS data and the scaled algae index classification method with NDVI. They hypothesized that Porphyra aquaculture along the North Jiangsu Shoal, where the Ulva macroalgae attaching onto the Porphyra rafts would be cleaned up after harvest and enter into the sea water, might be the origin of the green tide. ${ }^{21,22}$ However, because of resolution and weather condition restrictions, the precision of the green tide monitoring using MODIS data is low. ${ }^{20}$ Due to different measurement and comparison methods, scholars have hypothesized different ranges in error for green tide monitoring. Qiao et al. ${ }^{23}$ argued that the monitoring error is at the magnitude level, whereas Zhong et al. ${ }^{24}$ found that the error is $\sim 30 \%$ by comparing results extracted using the Chinese HJ-1 charge-coupled device (HJ-1 CCD) with the MODIS monitoring results.

Knowing the positional, areal coverage, and magnitude uncertainties for green tide monitoring using MODIS is a prerequisite for its use to estimate floating macroalgae blooms and to supply a service for the prevention of green tide. Given the variable patch size of a surface bloom (tens of meters to many kilometers), the potential error in using a large spatial sensor (e.g., MODIS with $250 \mathrm{~m}$ to $1 \mathrm{~km}$ resolution) to quantify the bloom must also be known. Owing to its advantages, such as flexibility, maneuverability, low cost, and high resolution, the unmanned aerial vehicle (UAV) can be used for real-time monitoring in the study area. ${ }^{25} \mathrm{In}$ addition, the oceanographic survey capabilities of the research vessel can perform accurate crosssectional studies of the green tide growth zones. However, few studies have quantified the MODIS green tide monitoring precision in terms of extent of areal coverage and position. To best accomplish this, accurate areal information and in-situ bloom properties are required. Therefore, this study combines an UAV equipped with an imaging system and cruise survey data to characterize the true distribution of the green tide. The objective of this study is to verify the detection efficiency for floating green tide using MODIS data. We compare MODIS monitoring green tide patches with the available field survey data and UAV image to verify whether the floating green tides were in the location detected by MODIS. Then, a green tide in 2016 was dynamically followed with MODIS Terra time series data to demonstrate the MODIS monitoring capability. In addition, the application of multisource data, including aerial imagery, in-situ observation data, and satellite images, is discussed.

\section{Materials and Methods}

\subsection{Overview of the Study Area}

The study area is in the South Yellow Sea at $32^{\circ}$ to $37^{\circ}$ north latitude and $119^{\circ}$ to $124^{\circ}$ east longitude. Within this area are several major coastal cities, including Lianyungang, Qingdao, Rizhao, and Weihai, as well as the North Jiangsu Shoal. As one of the primary green tide-stricken zones ${ }^{26}$ this area has seen the wild growth of green tide from May to August every year since 2008, causing losses to local tourism and aquaculture.

\subsection{Field Observations}

Three cruises employing the research vessel "Ke Xue San Hao," were conducted on May 17, May 19, and May 24, 2016, in the Yellow Sea. The weather conditions of May 17 and May 19 were both sunny but cloudy on May 24. Algal bloom records, including the locations, spatial extent, and observation times of Ulva patches, were collected from 96 stations (Fig. 1). Floating Ulva macroalgae were classified into seven magnitudes in accordance with the green tide distribution around each observation station based on the characteristics shown in Table 1 .

\subsection{Unmanned Aerial Vehicle Images and Processing}

DJI Inspire 1 systems were used in the study area. The devices included the Inspire 1 aircraft, two remote controllers (RC), and two mobile devices. A DJI X3(FC350) mounted below the 


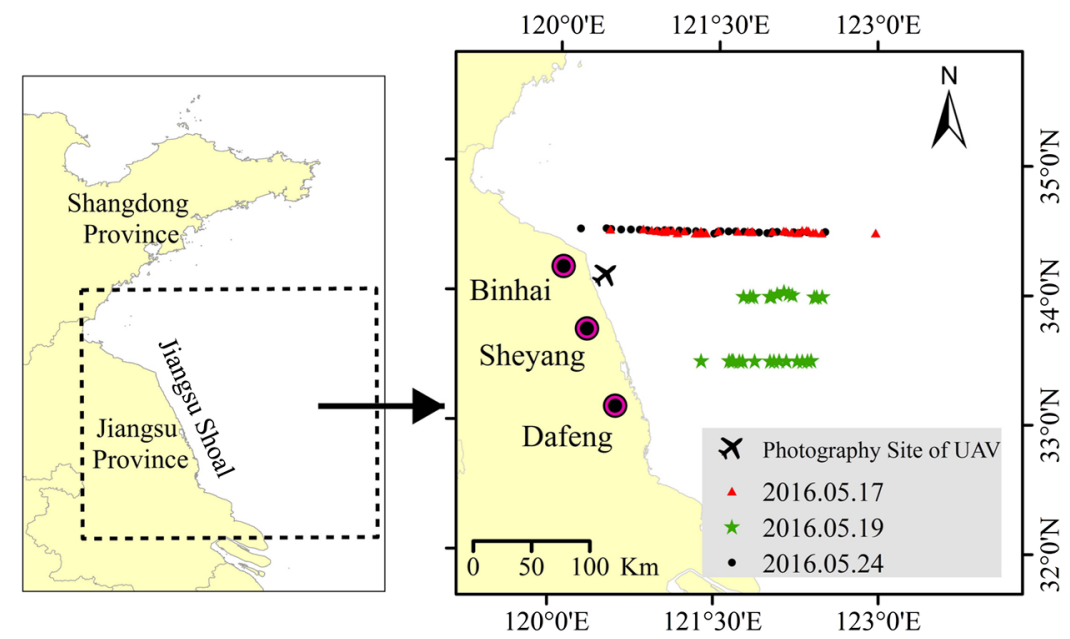

Fig. 1 Green tide observation stations and the site of the UAV photographed in the Yellow Sea in 2016.

Table 1 Magnitude levels of floating Ulva patches.

\begin{tabular}{ll}
\hline \hline Levels & \multicolumn{1}{c}{ Specification of corresponding Ulva algae patches } \\
\hline 0 & No green algae observed \\
1 & Less than five visible floating green algae patches; the magnitude is less than $1 \mathrm{~m} \times 1 \mathrm{~m}$ \\
2 & 5 to 10 visible floating green algae patches; the magnitude is less than $5 \mathrm{~m} \times 5 \mathrm{~m}$ \\
3 & $\begin{array}{l}10 \text { to } 20 \text { visible floating green algae patches that have formed a length over } 10 \mathrm{~m} \text { of a drifting } \\
\text { macroalgae banded structure }\end{array}$ \\
4 & $\begin{array}{l}\text { Greater than } 20 \text { visible floating green algae patches that have formed a length over } 20 \mathrm{~m} \text { of } \\
5\end{array}$ \\
6 & A floating green algae patch length greater than $500 \mathrm{~m}$ \\
\hline \hline
\end{tabular}

quadcopter aircraft was used to capture color (RGB) aerial images. The camera could capture color images with high longitudinal and lateral overlap controlled by RC and the mobile devices. The salvage ship of "Hai Zhuang Yuan," which was performing the Ulva salvage operation, provided a launching platform in the sea. Color aerial images, with a pixel size of $12 \mathrm{~cm}$, were taken vertically above the key Ulva-stricken zone at a height of $351 \mathrm{~m}$ on May 25 , 2016. The aerial photography location is shown in Fig. 1.

The UAV images and the associated georeferencing information provided by the GPS in the UAV were orthorectified and orthomosaiced into one image covering the regional area using a laptop with Pix4Dmapper. Subsequent spatial projection was processed in ArcGIS 10.2 with a reference coordinate system of WGS1984_UTM51N. In general, the RGB value of sea water was above 110 and the Red band was higher than the other two bands due to suspended sediment. By contrast, the RGB value of the green tide was below 100 and the red band was lower than the other two bands. Therefore, a threshold of the red band value was adopted to discriminate green tide using the raster color slices tool of the Environment for Visualizing Images (ENVI) 5.1. Finally, a red band value of 98 was selected to discriminate the green tide in the image, and the result was shown in Fig. 2(d).

\subsection{MODIS Data Processing}

MODIS Terra Level-1B data with a spatial resolution of $250 \mathrm{~m}$ and a geolocation product of MODIS (MOD03), provided by the NASA Ocean Biology Processing Group, were obtained 


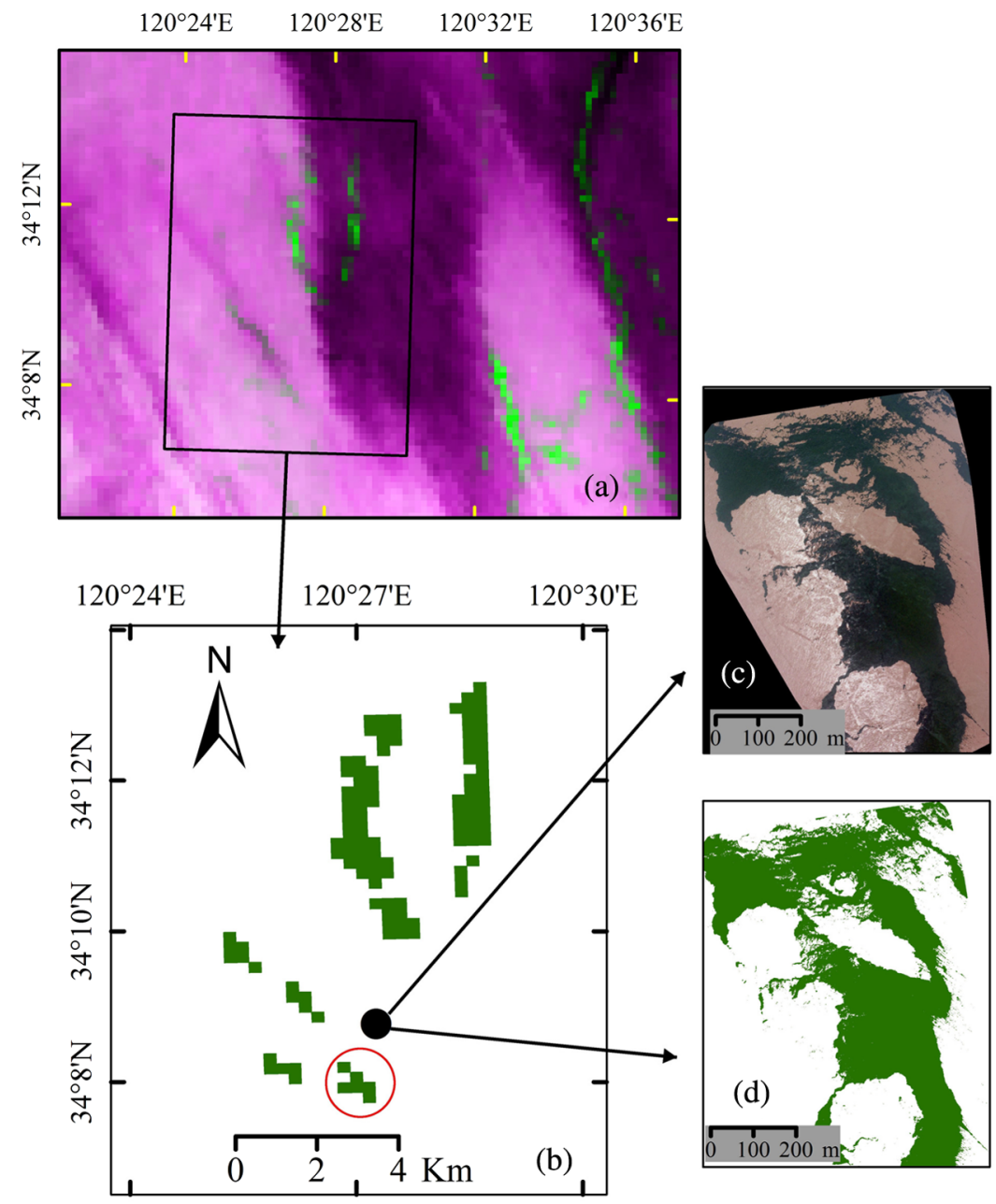

Fig. 2 Orthophoto using UAV and the distribution of the green tide:(a) composite false color image at the UAV photographed location; (b) Ulva patch distribution (green patches) extracted using MODIS and the location of the captured UAV images (black dot represents the location of the UAV photograph and the empty red circle represents the corresponding Ulva patch); (c) Orthophoto of Ulva patches captured using UAV; and (d) the distribution of Ulva patches extracted from the UAV orthophoto.

from the level-1 and atmosphere archive \& distribution system distributed active archive center website. ${ }^{27}$ A total of 46 cloudless or partly cloudy images were screened out for evaluation of MODIS performance in dynamic monitoring of the green tide from May to August in 2016. These images contain the entire growth cycle of the green tide in the Yellow Sea, including germination, development, bloom, and disappearance phases.

The water leaving radiance, where Ulva floats, is low at visible wavelengths, whereas it is high at near-infrared wavelengths. According to the spectral characteristics of green tide and seawater at visible to near-infrared wavelengths, the NDVI method can be used to extract green tide information: ${ }^{28}$

$$
\mathrm{NDVI}=\frac{B_{2}-B_{1}}{B_{2}+B_{1}}
$$

Either water leaving radiance or top of the atmosphere (ToA) can be applied to the NDVI calculation, but ToA was adopted in this paper because NDVI could also correct atmospheric and solar/viewing geometry effects to some extent with its normalization algorithm. ${ }^{29}$ Radiometric calibration and NDVI were computed in batches with ENVI. In detail, $B_{1}$ represents ToA at red wavelengths, corresponding to the first channel of MODIS $(0.620$ to $0.670 \mu \mathrm{m})$, and $B_{2}$ 
represents ToA at near-infrared wavelengths, corresponding to the second channel of MODIS $(0.841$ to $0.876 \mu \mathrm{m})$. The spatial resolution of both $B_{1}$ and $B_{2}$ equals $250 \mathrm{~m}$.

\subsection{Mapping the Floating Ulva Patches}

Ideally, the NDVI of Ulva patches is greater than zero; on the contrary, the NDVI of seawater is lower than zero. Hence, the threshold can be set to zero to discriminate green tide from seawater. However, some factors, e.g., sun glint and clouds, may interfere with the threshold. Therefore, the thresholds in this study were adjusted around zero and determined using the composite false color images. In the false color images, floating algae appeared jade green, while clouds appeared white, and seawater appeared purple or black, as shown in Figs. 2(a) and 3, suggesting a marked difference between the three features. Using this characteristic, we effectively identified and differentiated green tide and nongreen tide water after masking the land areas.

\subsection{Comparison Between MODIS Ulva Mapping and Field Observations}

The performance of MODIS for monitoring green tide was assessed by superimposing, and then comparing the satellite extracted location and magnitude with the corresponding field observation records, which were collected using the "Ke Xue San Hao" on May 17, May 19, and May 24,2016 . There is a time difference of dozens of minutes to several hours between satellite image collection and station observation. In addition, the green tide drifts spatially creating a mismatch between patches observed at the stations and the same patches in the satellite images. Therefore, buffer processing is adopted for the station observations. That is, we regard the green tide within a radius of each station as the result at the corresponding observation station. Based on the drift

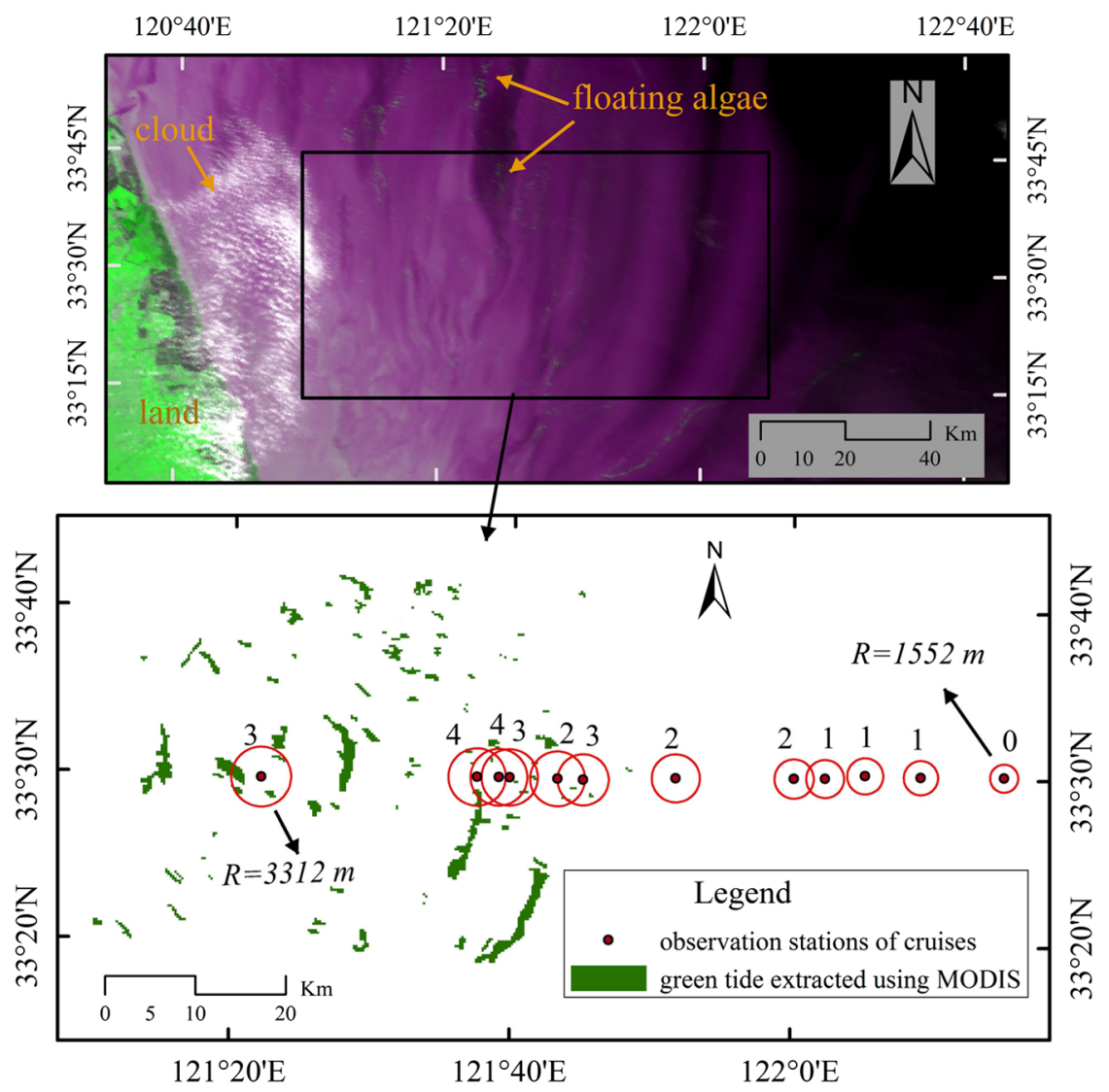

Fig. 3 Superimposition of green tide at the observation points with buffers (numbers above the dots represent the magnitude levels of green tide patches at the field observation sites) on May 19 , 2016. 

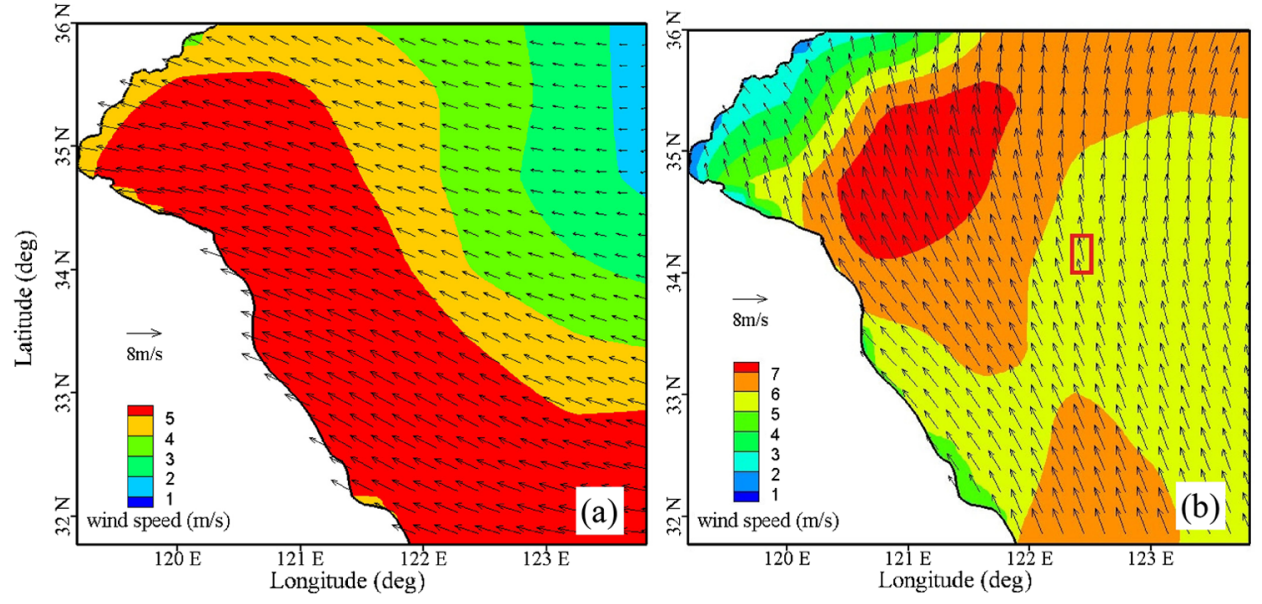

Fig. 4 SSW field of the study area: (a) SSW field on May 19, 2016 and (b) SSW field on May 25, 2016, empty red rectangle represents the location of the UAV photograph.

velocity of the green tide, we evaluated the buffer distance using the observation times and the sea surface wind (SSW) speed. According to previous studies, ${ }^{28,30}$ the buffer radius is defined by Eq. (2):

$$
R=0.023 * w * \Delta t
$$

where $R$ is the buffer radius, $w$ is the speed of the SSW, and $\Delta t$ is the time lag between the field observation and the satellite image acquired. The SSW were obtained from ERA-Interim datasets ${ }^{31}$ provided by the European Centre for Medium-Range Weather Forecasts. A case on May 19, 2016 is shown in Fig. 3, and the corresponding SSW is shown in Fig. 4(a).

\section{Results and Discussion}

\subsection{Cruise-Based Verification of MODIS Green Tide Monitoring}

A comparison between cruise observation data and intraday MODIS image interpretation (Table 2) shows that the monitoring (MODIS position relative to buffer position) of the green tide patch locations rose as the size of the patches increased. Except for the satellite image generated on May 24, which was affected by weather conditions, the Ulva monitoring ability for magnitude 1 bloom class $(<1 \mathrm{~m})$ was very low. Similarly, for cases with a magnitude 2 $(<5 \mathrm{~m})$ on May 17, May 19, and May 24, the detection efficiency was $33.33 \%, 50 \%$, and $0 \%$, respectively, which were higher than magnitude 1 , but too low to utilize in a monitoring

Table 2 Precision of the monitored green tide using MODIS.

\begin{tabular}{|c|c|c|c|c|c|c|}
\hline \multirow[b]{2}{*}{ Levels } & \multicolumn{2}{|c|}{ May 17} & \multicolumn{2}{|c|}{ May 19} & \multicolumn{2}{|c|}{ May 24} \\
\hline & Precision (\%) & Stations & Precision (\%) & Stations & Precision (\%) & Stations \\
\hline 0 & 100 & 5 & 100.00 & 9 & 50.00 & 4 \\
\hline 1 & 21.43 & 14 & 0 & 9 & 0 & 4 \\
\hline 2 & 33.33 & 3 & 50.00 & 4 & 0 & 6 \\
\hline 3 & 80.00 & 5 & 100 & 3 & 10.00 & 10 \\
\hline 4 & 100 & 5 & 100 & 2 & 25.00 & 12 \\
\hline 5 & - & - & - & - & 100 & 1 \\
\hline
\end{tabular}


application. However, the Ulva patches, whose sizes were tens of meters or longer (above magnitude 3), had high patch detection and discernment. Furthermore, the performance of the monitored green tide using MODIS was limited by weather conditions. Due to clouds and fog, the May 24 results showed that MODIS monitoring achieved a $<25 \%$ detection capability. Bad weather will reduce detection capability due to the influence on energy radiation. ${ }^{32}$

Previous work has documented that the spatial resolution of a satellite image is the primary limiting factor for detecting floating algae. ${ }^{20}$ With the resolution of $250 \mathrm{~m}$, the area of MODISderived Ulva patches was overestimated by an order of two times. ${ }^{28}$ By contrast, the compared areal extent varied by and order of magnitude indicating that MODIS images would underestimate the distribution of floating Ulva blooms. As shown in Table 1, the detection performance for small patches was low, while it was up to $100 \%$ for the station sites with no green algae patches. Therefore, the MODIS images tended to ignore the existence of small Ulva patches. One explanation could be that the information of the corresponding pixel in the MODIS image was primarily composed of the surrounding seawater if the Ulva patches were small, especially $<10 \mathrm{~m}$ in length. $\mathrm{Hu}$ et al. ${ }^{26}$ have also suggested that an algae slick $<5 \mathrm{~m}$ in width cannot be detected using $250 \mathrm{~m}$ MODIS FAI imagery. Similarly, Kutser ${ }^{33}$ indicated that the Hyperion (hyperspectral sensor) image with a spatial resolution of $30 \mathrm{~m}$ is not sufficient to detect narrow stripes of floating cyanobacterial scum, which appears extremely patchy and has the similar characteristics as Ulva when multispectral sensors are used.

\subsection{Unmanned Aerial Vehicles-Based Verification of MODIS Green Tide Monitoring}

As shown in Fig. 2(a), Ulva patches extracted using UAV, and MODIS images have a separation distance of $\sim 700 \mathrm{~m}$. Images using UAV were photographed at UTC 4:09 on May 25, whereas the interpreted MODIS image was obtained at UTC 2:40 on May 25. Considering the continuous mobility of floating Ulva derived by the wind field ${ }^{28}$ spatial mismatching was expected. In addition, as per the SSW data shown in Fig. 4(b) and Eq. (1), the UAV-derived patches correspond to the patch derived by MODIS in the red circle [Fig. 2(a)].

The UAV provided for the most accurate location and highest spatial resolution of patch size. The UAV derived patches shown in Fig. 2(c) covered an area of $0.06 \mathrm{~km}^{2}$, with a distribution density of $46.88 \%$. However, the area of the corresponding patch in the red circle (satellite derived) was $0.375 \mathrm{~km}^{2}$, which was 6.25 times that of the UAV-derived image. Analysis of other areas for the same Ulva patch indicated that the MODIS image had an abundance of mixed pixels consisting of seawater and floating algae information. In general, as shown in Figs. 2(b) and 5, the distributions of floating green algae patches were primarily branched and strip-shaped before beached, which was caused by the Langmuir circulation. ${ }^{34,35}$ Nevertheless, the MODIS-derived image depicted the patch distributions with grids of $250 \mathrm{~m}$ in width. Thus, the MODIS-derived area would overestimate the actual distribution area by several times. This could also explain why some studies showed that a difference in spatial resolutions could lead to a discrepancy of green algae bloom detection. ${ }^{24,28}$

\subsection{Monitoring Green Tide Using Multi-Source Data in the Yellow Sea}

The performance results above indicated that the MODIS images will tend to underestimate the existence of small algae patches but overestimate the area of floating Ulva blooms. However, we can conclude that MODIS images are very suitable for green tide monitoring because of its large coverage and high temporal resolution and the blooms, generally large patch size. Determining the location and area of floating Ulva blooms is important for creating management plans. ${ }^{20}$ The MODIS images can provide timely information on the location and area of green tide twice a day with coverage over the entire Yellow Sea, especially when floating biomass increased rapidly after mid-May. ${ }^{1}$ Furthermore, floating macroalgae blooms in the Yellow Sea can be traced back to $1999,{ }^{36}$ the year MODIS Terra was launched. Hence, MODIS data can provide a long time series of information for macroalgae blooms, which could help to understand the occurrence mechanisms and influence factors as well as creating management plans. 


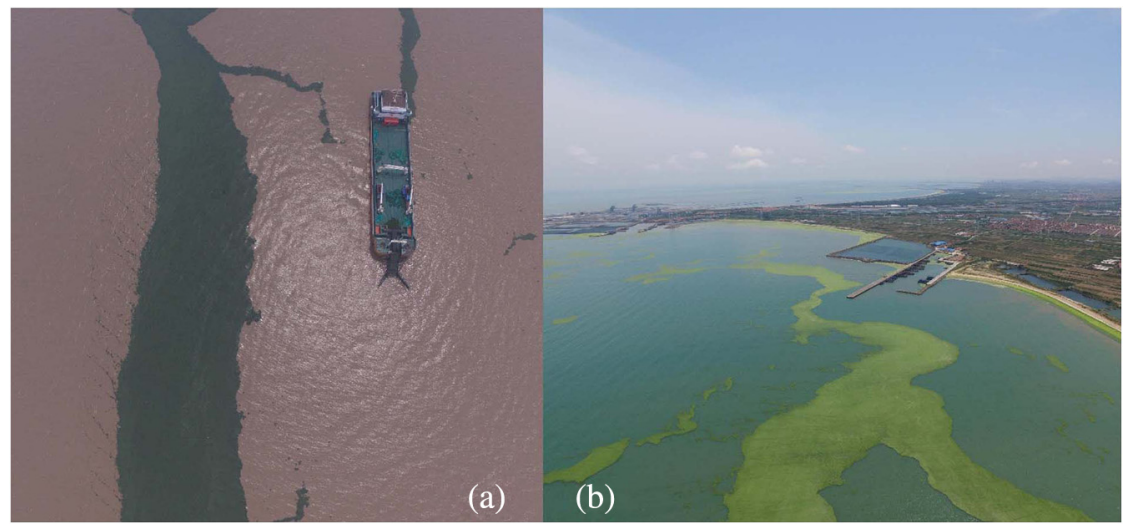

Fig. 5 UAV images of green tide at different locations: (a) UAV image taken above the waters near Jiangsu Shoal on May 25, 2016, and (b) UAV image taken along the coastline of Haiyang on July 6, 2016.

In order to demonstrate the MODIS monitoring capability, the green tide in 2016 was dynamically followed with MODIS Terra time series data. The northern boundaries of the event are shown in Fig. 6. The figures show that the floating blooms moved parallel to the coastline from Jiangsu Shoal, which first moved north, then northeast, and finally arrived at Rushan and Rongcheng via Qingdao. Ulva patches remained stable around Rizhao, Qingdao, Rushan, etc., due to the landforms of the Shandong Peninsula and the resistance from ocean currents. During this period, large-scale blooms accumulated on the shore and caused great losses to local tourism and the aquaculture industry. The results are consistent with previous studies. ${ }^{37,38}$

Figure 7 further shows that the tongue-shaped front edge of the floating Ulva blooms observed on June 1 morphed into a banded shape, as seen from Lianyungang to Qingdao on June 16. An explanation of the rapid increase in green tide may be the changes of light and temperature. ${ }^{39}$ The green tide was primarily distributed in muddy water before June 1 ,

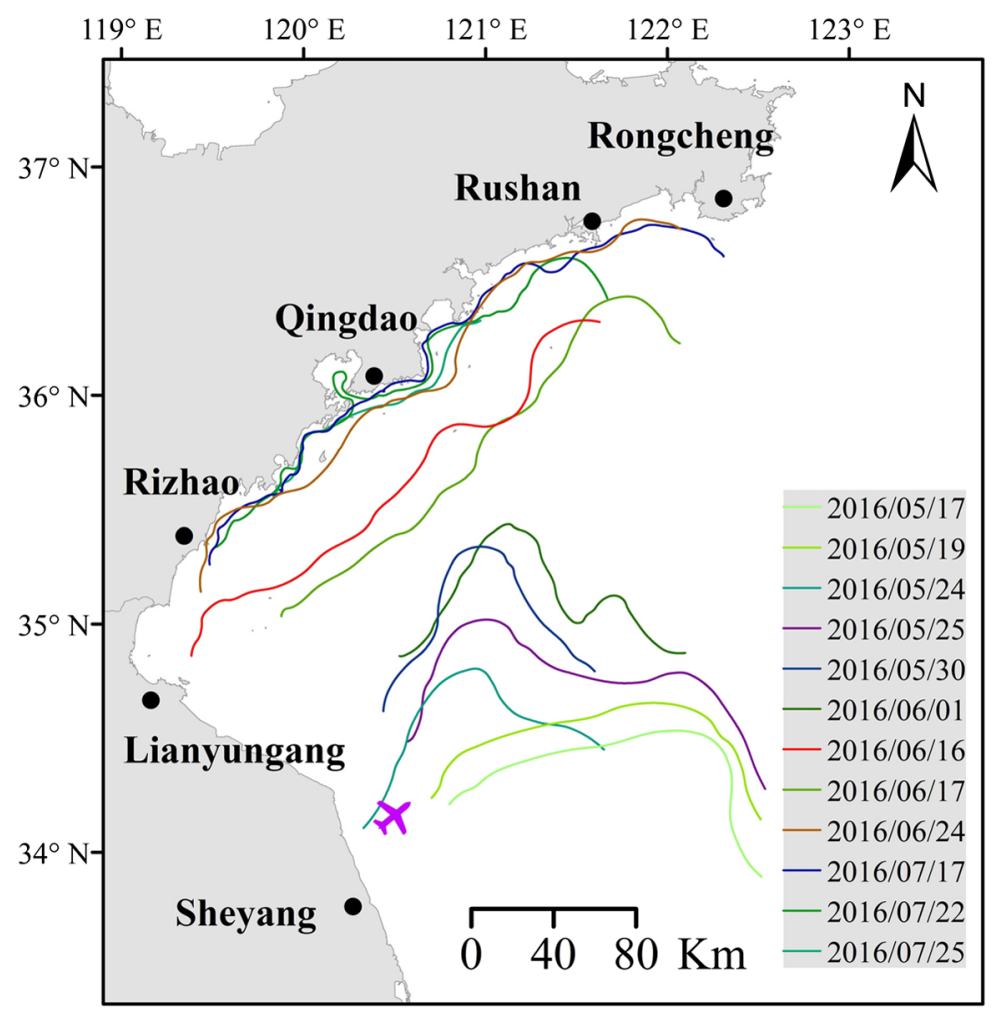

Fig. 6 The trend of the green tide northern boundaries in the Yellow Sea. 


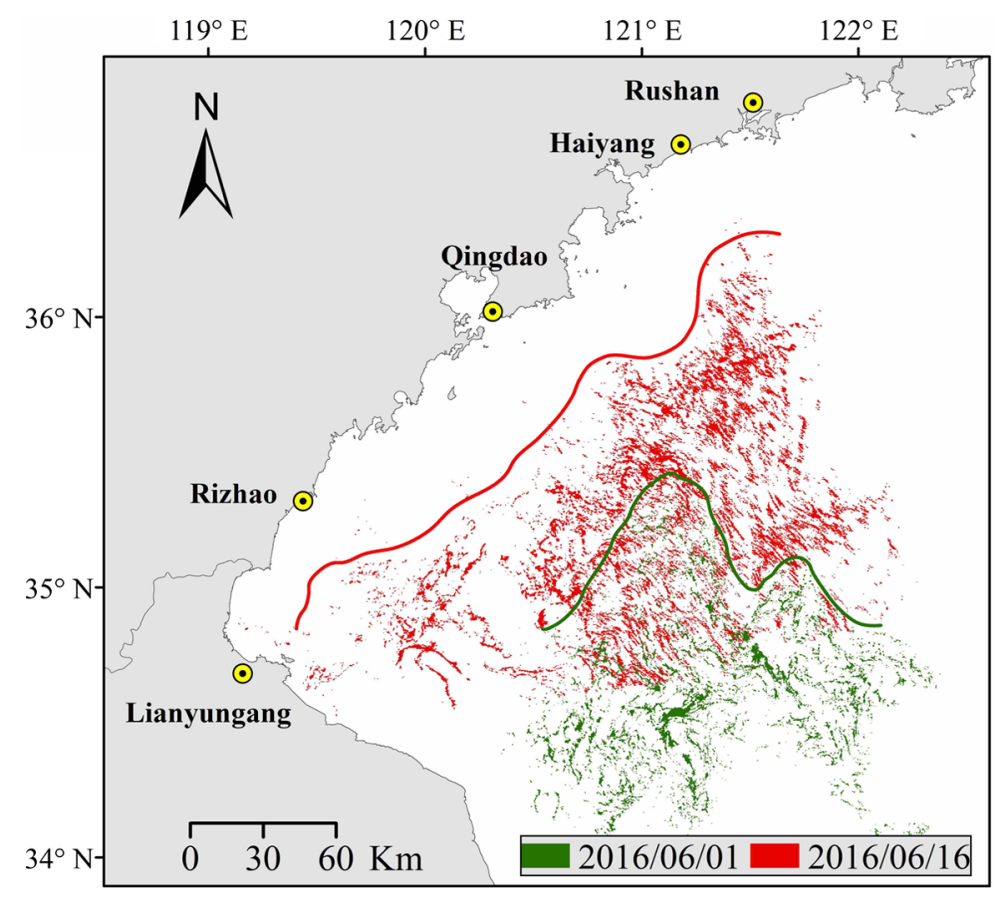

Fig. 7 The distribution of the green tide on June 1 and June 16 .

where it grew slowly due to the sunlight restrictions. Subsequently, the green tide drifted to clear water due to wind action and ocean currents and achieved explosive growth under favorable conditions, where the seawater temperature rose and light intensity increased. ${ }^{40,41}$ During this period, the daily growth rate of Ulva macroalgae can reach $10 \%$ to $37 \%$ under proper conditions. ${ }^{42}$

However, the satellite images with higher resolution and appropriate temporal resolution are still required to obtain detailed information of the green tide for decision making. Cui et al. ${ }^{28}$ suggested that HJ-1 CCD data is one of the most suitable data types for early detection and warning of green tide disaster, with a 30-m spatial resolution, 2 days for the revisiting period, and a 700-km swath width. Furthermore, GF-1 WFV data can be another appropriate choice for early detection, which has been used in green tide monitoring. ${ }^{43}$ It has a 2-day revisit period, 16-m spatial resolution, and $800-\mathrm{km}$ swath width. Moreover, the Sentinel-2A multispectral instrument (MSI) is the Landsat-like spatial resolution $(10$ to $60 \mathrm{~m}$ ) superspectral instrument of the European Space Agency, ${ }^{44}$ which has been proven useful for cyanobacterial scum detection. ${ }^{45}$ The Sentinel-2A MSI image includes 13 bands covering the visible and near infrared and shortwave infrared wavelength region. Specifically, $10-\mathrm{m}$ resolution with multispectral bands and three $20-\mathrm{m}$ resolution bands in the red-edge spectral domain, which is one of the best remote sensing-based descriptors of chlorophyll content, provide potential for green tide monitoring. ${ }^{46,47}$ Sentinel-2B, the second of the two-satellite Sentinel- 2 mission, will be launched in 2017, and the revisit time will decrease to 2 or 3 days in China (5 days at the equator). Hence, Sentinel-2 MSI and GF-1 WFV, as well as HJ-1 CCD, are recommended as the preferred satellite data for early observation and monitoring of the green tide in the Yellow Sea. With the multiple coverage capabilities, and with various spatial resolutions, the integration and fusion of data and detection probabilities can potentially greatly enhance our monitoring abilities over MODIS.

Continuous, real-time and high-precision acquisition of floating Ulva patches is very important for coping with the massive bloom. However, as indicated, few satellites in operation can meet the monitoring requirements. By contrast, UAVs have advantages, including flexibility, smaller dimensions, high spatial resolution, and low cost. With these advantages, the UAV can be the best tool for monitoring the green tide patches, especially at a regional scale. ${ }^{48,49}$ Specifically, the UAV was successfully used on the salvage ship during the Ulva bloom disaster in 2016. As shown in Fig. 8, the UAV could be highly utilized in optimizing the salvage operation route and assessing salvage operations. 


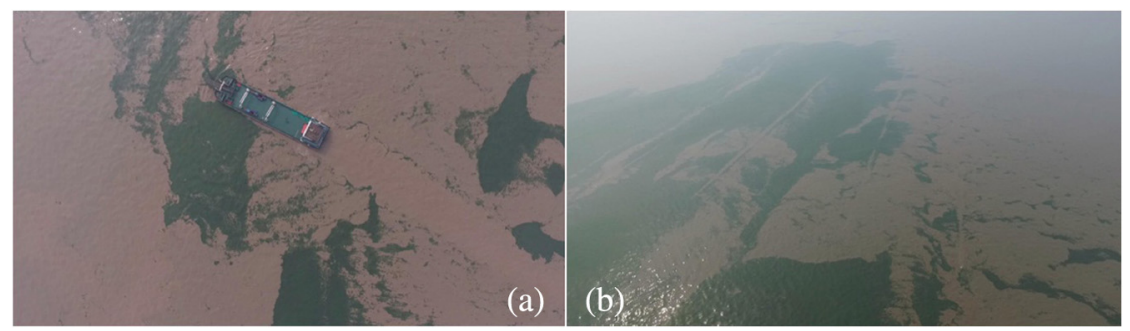

Fig. 8 UAV images for regional green tide monitoring: (a) UAV image used to optimize the salvage operation route and (b) UAV image used to assess the salvage operation.

\section{Conclusions}

For verifying the detection efficiency for green tide monitoring using MODIS, a comparison of MODIS green tide monitoring results with in situ observation data and UAV images was performed. The results showed that MODIS images could accurately detect the location of floating green algae patches but with higher uncertainty in size and cohesiveness when the patches were $<10 \mathrm{~m}$ in width. For the low spatial resolution and banded structure of drifting macroalgae, mixed pixels were prevalent in the MODIS images, which resulted in an overestimation of the distribution area by several folds. However, MODIS images were very suitable for green tide monitoring due to their high temporal resolution, long time series, and large coverage area. In addition, Sentinel-2 MSI, GF-1 WFV, and HJ-1 CCD may provide and even greater early green tide detection capability due to their high spatiotemporal resolution and broad swath width. In addition, the UAV could play an important role in monitoring regional green tides with the advantages of flexibility, smaller dimensions, high spatial resolution, and low cost. Therefore, comprehensive utilization of multisource data, including MODIS, Sentinel-2, GF-1 WFV, HJ-1 CCD, UAV and cruise survey data, would provide high precision information for green tide detection, forecast, and management.

\section{Acknowledgments}

This work was supported by the Aoshan Science and Technology Innovation Program of the Qingdao National Laboratory for Marine Science and Technology (2016ASKJ02), the Strategic Priority Research Program of the Chinese Academy of Sciences (XDA11020702), the Basic Special Program of the Ministry of Science and Technology (2014FY210600), and the Key Research Program of the Chinese Academy of Sciences (KZZD-EW-14).

\section{References}

1. S. Fan et al., "Temporal variation of green macroalgal assemblage on porphyra aquaculture rafts in the Subei Shoal, china," Estuarine Coastal Shelf Sci. 163, 23-28 (2015).

2. D. Y. Liu et al., "World's largest macroalgal bloom caused by expansion of seaweed aquaculture in China," Mar. Pollut. Bull. 58(6), 888-895 (2009).

3. T. A. Nelson et al., "Ecological and physiological controls of species composition in green macroalgal blooms," Ecology 89(5), 1287-1298 (2008).

4. D. G. Raffaelli, J. A. Raven, and L. J. Poole, "Ecological impact of green macroalgal blooms," Oceanogr. Mar. Biol. 125, 37-97 (1998).

5. J. K. Keesing et al., "Abiotic factors influencing biomass accumulation of green tide causing Ulva spp. on Pyropia culture rafts in the Yellow Sea, China," Mar. Pollut. Bull. 105(1), 88-97 (2016).

6. D. A. Lyons et al., "Macroalgal blooms alter community structure and primary productivity in marine ecosystems," Global Change Biol. 20(9), 2712-2724 (2014).

7. C. Wang, R. C. Yu, and M. J. Zhou, "Effects of the decomposing green macroalga Ulva (enteromorpha) prolifera on the growth of four red-tide species," Harmful Algae 16(2), 12-19 (2012). 
Xu et al.: Validation of MODIS-based monitoring for a green tide in the Yellow Sea with the aid...

8. X. H. Wang et al., "Economic cost of an algae bloom cleanup in China's 2008 Olympic Sailing Venue," Eos Trans. Am. Geophys. Union 90(28), 238-239 (2009).

9. Q. G. Xing et al., "Interpreting the progressive eutrophication behind the world's largest macroalgal blooms with water quality and ocean color data," Nat. Hazards 78(1), 7-21 (2015).

10. L. Kumar et al., "Review of the use of remote sensing for biomass estimation to support renewable energy generation," J. Appl. Remote Sens. 9(1), 097696 (2015).

11. Q. G. Xing et al., "World's largest macroalgal blooms altered phytoplankton biomass in summer in the Yellow Sea: satellite observations," Remote Sens. 7(9), 12297-12313 (2015).

12. R. M. Kudela et al., "Application of hyperspectral remote sensing to cyanobacterial blooms in inland waters," Remote Sens. Environ. 167, 196-205 (2015).

13. T. Kutser et al., "Monitoring cyanobacterial blooms by satellite remote sensing," Estuarine Coastal Shelf Sci. 67(1-2), 303-312 (2006).

14. M. B. Lyons, S. R. Phinn, and C. M. Roelfsema, "Long term land cover and seagrass mapping using landsat and object-based image analysis from 1972 to 2010 in the coastal environment of south east Queensland, Australia," ISPRS J. Photogramm. Remote Sens. 71(4), 34-46 (2012).

15. C. Petus et al., "Using modis data for understanding changes in seagrass meadow health: a case study in the great barrier reef (Australia)," Mar. Environ. Res. 98(4), 68-85 (2014).

16. S. Phinn et al., "Mapping seagrass species, cover and biomass in shallow waters: an assessment of satellite multi-spectral and airborne hyper-spectral imaging systems in moreton bay (australia)," Remote Sens. Environ. 112(8), 3413-3425 (2008).

17. A. Reinart and T. Kutser, "Comparison of different satellite sensors in detecting cyanobacterial bloom events in the Baltic sea," Remote Sens. Environ. 102(1), 74-85 (2006).

18. C. M. Hu and M. X. He, "Origin and offshore extent of floating algae in olympic sailing area," Eos Trans. Am. Geophys. Union 89(33), 302-303 (2008).

19. M. Q. Wu et al., "Research on the characteristics of Ulva. prolifera in shandong peninsula during 2008-2012 based on MODIS data," Spectrosc. Spectral Anal. 34(5), 1312-1318 (2014).

20. C. M. Hu, "A novel ocean color index to detect floating algae in the global oceans," Remote Sens. Environ. 113(10), 2118-2129 (2009).

21. J. K. Keesing et al., "Inter- and intra-annual patterns of Ulva green tides in the Yellow Sea during 2007-2009, their origin and relationship to the expansion of coastal seaweed aquaculture in China," Mar. Pollut. Bull. 62(6), 1169-1182 (2011).

22. D. Y. Liu et al., "The world's largest macroalgal bloom in the Yellow Sea, China: formation and implications," Estuarine Coastal Shelf Sci. 129, 2-10 (2013).

23. F. L. Qiao et al., "Drift characteristics of green macroalgae in the yellow sea in 2008 and 2010," Chin. Sci. Bull. 56(21), 2236-2242 (2011).

24. S. Zhong et al., "Error analysis on enteromorpha prolifera monitoring using modis data," Remote Sens. Inf. 28(1), 38-42 (2013).

25. A. Bhardwaj et al., "UAVs as remote sensing platform in glaciology: present applications and future prospects," Remote Sens. Environ. 175, 196-204 (2016).

26. C. M. Hu et al., "On the recurrent Ulva blooms in the Yellow Sea and East China Sea," J. Geophys. Res. Atmos. 115(C5), 640-646 (2010).

27. NASA Ocean Biology Processing Group, https://ladsweb.nascom.nasa.gov/search/ (15 September 2016).

28. T. W. Cui et al., "Satellite monitoring of massive green macroalgae bloom (GMB): imaging ability comparison of multi-source data and drifting velocity estimation," Int. J. Remote Sens. 33(17), 5513-5527 (2012)..

29. A. Bannari et al., "A review of vegetation indices," Remote Sens. Rev. 13(1), 95-120 (1996).

30. M. Bao et al., "Drifting trajectories of green algae in the western yellow sea during the spring and summer of 2012," Estuarine Coastal Shelf Sci. 163, 9-16 (2015).

31. "European centre for medium-range weather forecasts," http://apps.ecmwf.int/datasets/data/ interim-full-daily/levtype $=\mathrm{sfc} /(21$ December 2016). 
32. A. P. Trishchenko, J. Cihlar, and Z Li, "Effects of spectral response function on the surface reflectance and NDVI measured with moderate resolution sensors," Remote Sens. Environ. 81(1), 1-18 (2002).

33. T. Kutser, "Quantitative detection of chlorophyll in cyanobacterial blooms by satellite remote sensing," Limnol. Oceanogr. 49(6), 2179-2189 (2004).

34. F. L. Qiao et al., "Banded structure of drifting macroalgae," Mar. Pollut. Bull. 58(12), 17921795 (2009).

35. S. A. Thorpe, "Spreading of floating particles by langmuir circulation," Mar. Pollut. Bull. 58(12), 1787-1791 (2009).

36. Q. G. Xing and C. M. Hu, "Mapping macroalgal blooms in the Yellow Sea and East China Sea using HJ-1 and Landsat data: application of a virtual baseline reflectance height technique," Remote Sens. Environ. 178, 113-126 (2016).

37. V. Smetacek and A. Zingone, "Green and golden seaweed tides on the rise," Nature 504(7478), 84-88 (2013).

38. Y. B. Son et al., "Tracing floating green algae blooms in the yellow sea and the east china sea using goci satellite data and lagrangian transport simulations," Remote Sens. Environ. 156, 21-33 (2015).

39. J. Wang et al., "Ecological factor research on the growth and induction of spores release in Enteromorpha prolifera (chlorophyta)," Mar. Sci. Bull. 26(2), 60-65 (2007).

40. W. Qing et al., "An adjustment mechanism to high light intensity for free-floating Ulva in the yellow sea," J. Shanghai Ocean Univ. 25(1), 97-105 (2016).

41. E. Waigmann, "Observations on the effect of salinity and photon fluence rate on the induction of sporulation and rhizoid formation in the green alga Enteromorpha prolifera (Muller) J. Agardh (Chlorophyta, Ulvales),' Fish. Sci. 68(6), 1182-1188 (2002).

42. Z. Y. Liang et al., "A preliminary study of the enteromorpha prolifera drift gathering causing the green tide phenomenon," Period. Ocean Univ. China 38(4), 601-604 (2008).

43. X. Y. Zheng et al., "Remote sensing monitoring of green tide in the Yellow Sea in 2015 based on GF-1 WFV data," Proc. SPIE 9975, 99750L (2016).

44. H. V. D. Werff and F. V. D. Meer, "Sentinel-2A MSI and Landsat 8 OLI provide data continuity for geological remote sensing," Remote Sens. 8(11), 883 (2016).

45. European Space Agency, "Sentinel-2 catches eye of algal storm," (2015), http://www.esa. int/Our_Activities/Observing_the_Earth/Copernicus/Sentinel-2/Sentinel-2_catches_eye_of_ algal_storm (25 November 2016).

46. P. J. Curran, "Exploring the relationship between reflectance red edge and chlorophyll content in slash pine," Tree Physiol. 15(3), 203-206 (1990).

47. A. Fernández-Manso, O. Fernández-Manso, and C. Quintano, "Sentinel-2A red-edge spectral indices suitability for discriminating burn severity," Int. J. Appl. Earth Obs. Geoinf. 50, 170-175 (2016).

48. J. A. J. Berni et al., "Thermal and narrowband multispectral remote sensing for vegetation monitoring from an unmanned aerial vehicle," IEEE Trans. Geosci. Remote Sens. 47(3), 722-738 (2009).

49. U. Ozdemir et al., "Design of a commercial hybrid vtol UAV system," J. Intell. Rob. Syst. 74(1), 371-393 (2014).

Fuxiang $\mathbf{X u}$ is a research assistant at the Yantai Institute of Coastal Zone Research, Chinese Academy of Sciences, Beijing, China. He received his MS degree in soil science from the Fujian Agriculture and Forestry University, Fuzhou, China, in 2015 and is currently pursuing his PhD at the University of Chinese Academy of Science, Yantai, China, in environmental science. His current research interests include environment remote sensing and marine disaster monitoring.

Zhiqiang Gao received his MS degree from the Institute of Xinjiang Geography, Chinese Academy of Science, Urumqi, China, in 1993 and his $\mathrm{PhD}$ from the Institute of Remote Sensing Application, Chinese Academy of Science, in 1998, both in mapping and geographic information system. Now, he is a professor with the University of Chinese Academy of Science, Beijing, China and a research scientist with the Yantai Institute of Coastal Zone Research, 
Chinese Academy of Science. His research interests include remote sensing application and model simulation in coastal zone.

Weitao Shang received his MS degree in cartography and geographic information system from Lanzhou Jiaotong University, Lanzhou, China, in 2014. Now, he is an engineer at Yantai Institute of Coastal Zone Research, Chinese Academy of Science. His current research interests include UAV remote sensing and WebGIS development.

Biographies for the authors are not available. 